\title{
Marc Winter \\ Lust und Schmerz als sexuelle Konzepte? \\ Warum es im vormodernen China keinen \\ Sadomasochismus gab
}

Lust und Schmerz sind sinnliche Empfindungen, die namentlich in den sexuellen Praktiken des Sadismus und Masochismus eng miteinander verknüpft sind und unter dem beide Aspekte einschliessenden Begriff der Algolagnie, des sexuellen Lustempfindens beim Erleiden oder Zufügen von Schmerzen, zusammengefasst werden. Aus klassisch-sinologischer Perspektive interessiert mich die Frage: Welche Rolle spielen Schmerz und Lust am Schmerz im Rahmen des Diskurses über Sexualität in der vormodernen chinesischen Gesellschaft? Dabei wird ein modernes westliches psychologisches Konzept kulturübergreifend auf eine vormoderne Gesellschaft angewandt in der Hoffnung, Aspekte dieser Gesellschaft sichtbar zu machen, die sich sonst möglicherweise einer wissenschaftlichen Einschätzung entziehen, weil sie beispielsweise im Binnendiskurs einer Kultur gar nicht thematisiert würden. Die nachfolgende Untersuchung stützt sich auf literarische, historische und philosophische Quellen sowie auf Sex-Handbücher, die im kaiserzeitlichen China zirkulierten, um Äusserungen der vormodernen chinesischen Gesellschaft zu beschreiben, die mit dem lustvollen Erleiden oder Zufügen von Schmerzen zusammenhängen. Alleine aufgrund des Umfangs der vorliegenden Diskussion ist offensichtlich, dass eine solche Darstellung weder umfassend noch erschöpfend sein kann, sondern lediglich eine grobe Skizze. Ich argumentiere im Folgenden nicht psychoanalytisch, sondern kulturanalytisch. 
162 Marc Winter: Lust und Schmerz als sexuelle Konzepte?

Meinen Überlegungen liegt die grundsätzliche Auffassung zugrunde, dass Sexualität stark kulturell geprägt ist. Entgegen einem biologistischen Ansatz, der Sexualität als anthropologische Konstante interpretiert, scheint mir evident, dass Sexualität eine Kulturleistung ist, die - wenn auch aufbauend auf biologischen Gegebenheiten - von einem bestimmten Körperverständnis und von konkreten moralischen Normen geprägt ist, von der Beziehung zwischen den Geschlechtern und von bestimmten religiösen und philosophischen Vorstellungen.

Gleichwohl ging ich von der Vermutung aus, dass es in China Phänomene von Algolagnie geben würde, die mit denen im Westen vergleichbar sind, aber ich sah mich zu meiner Überraschung getäuscht. Ausgehend von der Hypothese, dass Sadismus und Masochismus Elemente oder Varianten der breiten Palette menschlicher Sexualität sind, die in jeder Kultur vorkommen, musste ich bald feststellen, dass sich für China so gut wie keine Hinweise hierfür finden liessen. Das bedeutet allerdings nicht, dass es nicht einzelne Personen oder Paare gab, die sadistische oder masochistische Sexualität praktizierten. Die Tatsache, dass sich so gut wie kein Hinweis auf Sadismus oder Masochismus finden liess, bedeutet aber, dass kein Diskurs hierüber stattfand, dass diese sexuellen Vorlieben gesellschaftlich nicht erörtert, dass keine Texte dazu geschrieben wurden. Mit anderen Worten, Sadomasochismus wurde vielleicht vereinzelt praktiziert, aber es wurde kulturell und literarisch kaum thematisiert.

\section{Terminologie und literarische Quellen}

Die Annahme, dass diese Konzepte und Spielarten der chinesischen Sexualität fremd waren, wird bereits durch die Terminologie bestätigt. Es existiert kein Begriff aus der chinesischen Antike oder dem Mittelalter, der ein solches Verhalten beschreibt. Vielmehr sind die verwendeten Begriffe bezeichnend, weil es offenkundige Neologismen sind: Masochismus wird als 受虐癖 shounüepi oder 
163 Marc Winter: Lust und Schmerz als sexuelle Konzepte?

shounüekuang 受虐狂 übersetzt, Sadismus als 施虐癖 shinüepi oder shinuekuang 施虐狂. Die Ausdrücke sind parallel gebildet. Die Wörter pi beziehungsweise kuang bezeichnen ein krankhaftes Verlangen, nüe heisst Grausamkeit, und die Verben shi und shou bedeuten ausüben beziehungsweise erdulden. Die chinesischen Ausdrücke für Sadismus und Masochismus sind also zu übersetzen als: die Sucht, grausame Handlungen an einem Menschen vollziehen beziehungsweise das empfangende Objekt solcher grausamen Handlungen zu sein. Die Ausdrücke sind mit ihrer nüchternen Betonung des technischen Vorgangs, der die Lustempfindung an der ausgeübten oder empfangenen ausblendet, Übersetzungen eines kulturfremden Konzeptes.

Was Belegstellen in der Literatur angeht, die man mit Sadismus oder Masochismus in Verbindung bringen könnte - natürlich unter Verwendung anderer Begriffe als der eben genannten -, so gibt es nur sehr wenige Hinweise, die allerdings alles andere als eindeutig sind. In der song-zeitlichen Anekdotensammlung Hou qing lu 侯鯖錄 des Zhao Delin 趙德麟 (Zhao Lingzhi 趙令畤 1061-1134) ist eine Episode verzeichnet, die schon von Robert van Gulik erwähnt wurde. ${ }^{1}$ Demzufolge lebte während der Song-Dynastie im 11. Jahrhundert ein Kreismagistrat namens Lü Shilong in Xuanzhou (Xuan chengshou Lü Shilong 宣城守吕士隆). Dieser ging rigoros gegen die Prostitution in seinem Landkreis vor. Er liess Prostituierte regelmässig verhaften und peitschte sie aus, und es wird dort berichtet, dass er Gefallen daran fand. Später verliebte sich Lü Shilong in eine Kurtisane namens Lihua 麗華 aus Hangzhou und heiratete sie. Der Text unterscheidet hier zwischen der Prostituierten jinü 妓女 und der Kurtisane chang 娼, beide Begriffe bezeichnen unterschiedliche «Klassen» von käuflichen Frauen. Die Kurtisane ist eine höher klassifizierte Prostituierte, eine Unterhalterin und Gesellschafterin, die auch Gedichte rezitieren und ein Instrument spielen kann.

1 Robert Hans van Gulik: Sexual Life in Ancient China. A Preliminary Survey of Chinese Sex and Society from ca. 1500 B.C. till 1644 A.D. = Zhongguo gudai fangnei kao 中國古代房內考, Leiden 1974, 160-161. 
164 Marc Winter: Lust und Schmerz als sexuelle Konzepte?

Eines Tages schickte Lü Shilong sich wiederum an, eine Prostituierte $\mathrm{zu}$ verprügeln, als diese weinend seine Knie umschlang und in mit den folgenden Worten anflehte:

$\begin{array}{lll}\text { 某不敢避 } & \text { Mou bu gan bi } & \text { Ich wage es nicht, um Schonung } \\ \text { 杖, 但恐 } & \text { zhang, dan kong } & \text { vor dem Stock zu betteln, aber ich } \\ \text { 新到某人 } & \text { xin dao mou ren } & \text { fürchte, dass jene, welche eben erst } \\ \text { 者, 不安 } & \text { zhe, bu an ci er } & \begin{array}{l}\text { hierhergekommen ist, dies wohl } \\ \text { nicht so ruhig [ertragen] könnte. }\end{array} \\ \text { 此耳 } & & \end{array}$

Diese Episode wird aufgefasst als ein Argument, wonach die Prostituierte wusste, dass Lü Shilong Prügelstrafen zu verhängen pflegte, vielleicht auch, dass er Lust dabei empfand. Aber sie erinnerte ihn daran, dass seine neue Frau Lihua vielleicht seine Grausamkeit abstossend finden und ihm deshalb die kalte Schulter zeigen könnte. Lü habe darauf lachend ein Einsehen gehabt und von der Strafe abgesehen.

Später verfasste Mei Xiaochen 梅尧臣 (1002-1060) zur Erklärung dieser Episode das folgende Gedicht mit dem Namen «Nicht die Ente schlagen!»:

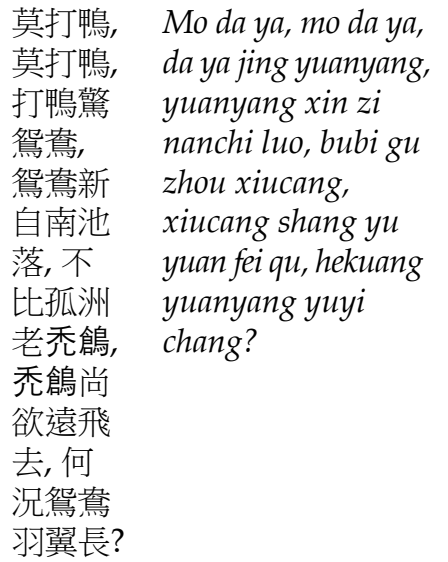

Schlage nicht die Enten, schlage nicht die Enten, denn die Enten zu schlagen könnte die Mandarinenente erschrecken. Die Mandarinenente hat sich - eben erst vom südlichen Weiher kommend - hier niedergelassen, sie ist kein Vergleich mit den altbekannten kahlköpfigen Schwarznackenpirol dieser vereinzelten Insel im Strom. Die kahlköpfigen Schwarznackenpirole wollen noch weit hinweg fliegen, aber was nützt es der Mandarinenente noch, lange Flügel zu haben? ${ }^{2}$

2 Van Gulik erwähnt die Episode, bei Liu Dalin gibt es die Originaltexte, die Übersetzungen stammen von mir. R. H. van Gulik: Sexual Life in Ancient China, 160-161; Liu Dalin: 刘达临 1993; Zhongguo Gudai Xing Wenhua $=$ The Sex Culture of Ancient China 中国古代性文化, Yinchuan 1993, 314. 
165 Marc Winter: Lust und Schmerz als sexuelle Konzepte?

Diese Episode wird in der sexologischen Literatur zum Alten China als Evidenz für die Existenz von Sadomasochismus angesehen. Die Mandarinenente, ein Symbol ehelicher Treue, die ihre Flügel ja nicht mehr braucht, kann vielleicht den Rohrstock des Ehemannes zu spüren kriegen, aber die normalen Enten sind mit Narben oder sonstigen Zeugnissen der Behandlung durch Lü Shilong fürs Leben gezeichnet.

Im ming-zeitlichen erotischen Roman Jinpingmei 金瓶梅 (Pflaumenblüten in Goldener Vase) ist wiederholt die Rede davon, dass der sexbesessene Protagonist Xi Men vor dem Geschlechtsverkehr drei brennende Kegel Räucherstäbchen auf dem nackten Körper der Frau platzierte: einen Kegel zwischen den Brüsten, einen auf dem Unterleib und einen auf dem Venushügel. Die Frau ist mit dieser Praxis einverstanden, ja es erhöht sogar ihre sexuelle Erregung. ${ }^{3}$ Die Szenen führen nicht zur geschlechtlichen Vereinigung, sondern als die Räucherkegel heiss werden und die Frau es nicht mehr erträgt, lässt Xi Men sie Treueschwüre sprechen, bei denen es nicht um Sex geht.

In den Sex-Handbüchern sind Hinweise auf Kratz- oder Bisspraktiken rar, anders als in der einschlägigen indischen Literatur, wo Sanskrit-Handbücher Schlagen, Beissen und Kratzen in grosser Ausführlichkeit beschreiben. Masochistische Praktiken sind in der altchinesischen ebenso wenig klar auszumachen. Robert van Gulik zitiert hingegen den Fall des berühmten Polyhistors Shen Kuo 沈括 (1030-1094). ${ }^{4}$ Dieser, so berichtet Zhu Yu 朱或 ( 1100) im Pingzhou Ketan 萍洲可谈, habe eine Frau mit Nachnamen Zhang geheiratet, die ihn geschlagen, geprügelt und ihm Barthaare ausgerissen habe, worüber er sich aber nie beklagt habe. Nachdem die Frau gestorben war, sei er untröstlich gewesen und wollte sich ertränken. Dieser Fall wird als das eindeutigste Zeugnis von Masochismus gewertet, das in der chinesischen Literatur zu finden ist.

3 R. H. van Gulik: Sexual Life in Ancient China, 161; Xia Wei 夏薇: “Xing shi yin yuan zhuan» yanjiu 《醒世姻缘传》研究 (Forschung zum «Xing shi yin yuan zhuan»), Beijing 2007, 65, zit. Kapitel 78.

$4 \quad$ R. H. van Gulik: Sexual Life in Ancient China, 162. 
166 Marc Winter: Lust und Schmerz als sexuelle Konzepte?

Neben dem erwähnten Roman Jinpingmei wurden in der letzten kaiserlichen Dynastie Qing (1644-1911) weitere Texte zu diesem Thema geschrieben - wie beispielsweise das Sex-Handbuch Chongming Manlu 虫鸣漫录 (Aufzeichnungen von Amüsantem [aus dem Studio] des Insektenzirpens) von Cai Hengzi 菜德子. Der wohl bekannteste Text in dieser Hinsicht ist der Roman Xingshi yinyuan zhuan 醒世姻 缘传, was so viel bedeutet wie Geschichten von Eheleuten, die vom Schicksal zusammengeführt wurden und die die Welt aufrütteln werden. ${ }^{5}$ Dieses Buch mit 100 Kapiteln ist die wichtigste Quelle für alle Arten von Sexualpraktiken. Es ist eine Sammlung von Geschichten zum Eheleben und zu Zweierbeziehungen. In den Geschichten werden Dinge erörtert, die sonst nirgends thematisiert werden, wie beispielsweise Gattenmord oder weibliche Untreue; es finden sich darunter auch Episoden über die Grausamkeit zwischen Eheleuten, die etwa darüber berichten, wie ein Mann seine Frau in den Tod treibt oder eine Ehefrau ihren unterwürfigen Mann quält. Gleichwohl bin ich skeptisch, diesen Text als Zeugnis für Algolagnie zu bezeichnen. Grund für meine Skepsis ist erneut, dass nur das Leiden und der Schmerz thematisiert werden, nicht die Lust daran. Vielmehr geht es im Xingshi yinyuan zhuan um karmische Vergeltung.

Xia Wei grenzt den Entstehungszeitraum des Werkes auf die Jahre 1726-1792 ein. ${ }^{6}$ Traditionell wird es einem Autor zugeschrieben, der seinen Namen hinter dem Pseudonym Xi Zhousheng verbarg 西周生. Es gibt mehrere Theorien über die Autorschaft. Hu Shi 胡適 (1891-1962) vertrat die Ansicht, das Buch sei von Pu Songling 蒲松齡 (1640-1715) verfasst worden, dem berühmten Autor der Sammlung von Geistergeschichten Liaozhai Zhiyi 仰齋誌異: hier gibt es Begegnungen mit Untoten und Gespenstern wie mit Fuchsfeen, das heisst in Frauengestalt verwandelten Füchsen, die Männern Böses wollen und antun. Allerdings blieb $\mathrm{Hu}$ Shi mit seiner Zuweisung

5 Xi Zhousheng 西周生: Xingshi yinyuan zhuan 醒世姻緣傳, Beijing 1993.

6 Xia Wei 夏薇: «Xing Shi Yin Yuan Zhuan» zhong de lüechi xianxian《醒世 姻缘传》中的虐恋现象》 (Das Phänomen des Sadomasochismus im «Xing shi yin yuan zhuan»), in: Zhongguo gudai xiaoshuo yanjiu = Studies in Chinese Classical Fiction 2 (2006) 203-216. 
weitgehend alleine; aus meiner Sicht ist sein Argument wenig überzeugend, weil es ausschliesslich auf der Tatsache aufbaut, dass beide Werke Dinge thematisieren, die sonst Tabu sind. ${ }^{7}$

In Helwig Schmidt-Glintzers Geschichte der chinesischen Literatur wird das Werk wie folgt zusammengefasst und ohne Hinweis auf einen sexuellen Kontext beurteilt:

Der Roman Xingshi yinyuan zhuan handelt von einer glücklosen Heirat, der Vergeltung an dem Ehemann für seine mitleidslose Erlegung eines Fuchses und für die schlechte Behandlung, die er seiner Frau in einem früheren Leben hatte zuteilwerden lassen. Alles Unheil leitet der Verfasser des Romans davon ab, dass nicht die wesentliche Voraussetzung, nämlich die richtige Partnerin zur Frau zu haben, erfüllt ist. Danach ist eine glückliche Ehe das Zeichen für ein gutes karmisches Schicksal. [...] Die ersten 22 Kapitel des aus vielen Episoden zusammengesetzten Romans kreisen um die Hauptfigur Chao Yuan und dessen Familie in einer Stadt der Provinz Shandong. Der zweite und längere Teil des Romans, Kapitel 23 bis Kapitel 100, handelt von der Familie Di, in die Chao Yuan wiedergeboren wird, und von der Rache der mit ihm verheirateten Frau, die just die Reinkarnation des von Chao Yuan in seinem vorherigen Leben getöteten Fuchses ist. Seine zweite Frau ist die wiedergeborene, von ihm schlecht behandelte Frau aus seinem früheren Leben. Doch erst nach zahlreichen Verstrickungen wird der Held geläutert und kann schliesslich, nachdem er die buddhistischen Gebote für Laien befolgt, mit der zweiten Frau - die erste ist inzwischen verstorben in Frieden zusammenleben. Mit solcher Kritik an bestimmten Verhaltensweisen, insbesondere an der Missachtung von Frauen, wird zugleich eine allgemeine Kritik an dem herrschenden Wertesystem geübt. ${ }^{8}$

In ähnlichem Sinne und ebenfalls ohne Hinweis auf sadomasochistische Praktiken beschreibt Clemens Treter die Hauptfigur des Romans Chao Yuan:

Chao Yuan [...] der Sohn eines durch Betrug an die Macht gelangten Beamten, nimmt sich eine Geliebte, vernachlässigt seine Frau und treibt diese schliesslich durch falsche Anschuldigungen sogar in den Selbstmord. ditional Chinese Literature, Taipei 1986, 564. 3000jährige Entwicklung der poetischen, erzählenden und philosophischreligiösen Literatur Chinas von den Anfängen bis zur Gegenwart, Bern, München, Wien 1990, 466. 
168 Marc Winter: Lust und Schmerz als sexuelle Konzepte?

[Seine Wiedergeburt] Di Xichen hingegen [...] steht eindeutig unter dem Pantoffel sowohl seiner Ehefrau als auch einer später heimlich geheirateten Konkubine. Der Roman reiht eine ungeheure Fülle überaus realistisch geschilderter Eheszenen aneinander, die oft noch zusätzlich durch Ereignisse in Nachbarsfamilien indirekt kommentiert werden. ${ }^{9}$

Im Xingshi yinyuan zhuan wird - jenseits vom buddhistischen Anliegen für kosmische Gerechtigkeit - eine Welt geschildert, die aus den Fugen geraten ist, was dem literarischen Stil der Zeit entspricht. Der von Metaphern und intertextuellen Anspielungen durchdrungene Roman enthält - zumindest aus der Sicht des modernen Lesers keine Situation, die erotisch aufgeladen ist. Das Buch ist eher moralisch; es spielt auf die asymmetrische Natur einer Beziehung zwischen einem dominantem und einem submissiven Partner an, indem es sehr allgemein auf die Naturgewalten Himmel und Erde und auf ein Ungleichgewicht zwischen ihnen referiert. Es gibt auch keine detaillierten Folterszenen, sondern es wird die Möglichkeit erörtert, dass es in der Liebesbeziehung zwischen Mann und Frau auch gewalttägige «Gewitter» und Unausgeglichenheiten zwischen yin und yang geben kann. Das Buch ist daher kaum zur erotischen Literatur zu zählen.

Zusammenfassend ist festzuhalten, dass es durchaus Aufzeichnungen über Algolagnie als sexuelles Stimulans gibt, wenn auch nur in der späten Kaiserzeit und nicht als Tatsachenberichte, sondern in literarischen Texten. Zudem sind diese Quellen ausgesprochen dürftig - eine Begeisterung für dieses Genre und eine entsprechend hohe Nachfrage scheint sich nicht eingestellt zu haben.

\section{Erklärungsversuche}

Erklärungsversuche sind zwangsläufig spekulativ und müssen unter Einbeziehung kulturdefinierender Faktoren unternommen werden. Zwangsläufig deshalb, weil die Quellenlage keine eindeutigen

$9 \quad$ Reinhard Emmerich (Hg.), Hans van Ess, Raoul David Findeisen, Martin Kern, Clemens Treter (Mitarb.): Chinesische Literaturgeschichte, Stuttgart, Weimar 2004, 260. 
Belege zulässt. $\mathrm{Zu}$ den zitierten literarischen Texten gibt es keine Paratexte mit entsprechenden Aussagen. Das erwähnte Ungleichgewicht zwischen den Urkräften scheint mir bedeutsam zu sein für die Erklärung, wieso Lust und Schmerz als sexuelle Konzepte in der chinesischen Kultur nicht gut zusammengingen. Während Sadismus im Sinne von Lust an Grausamkeit auch in China ein verbreiteter und etablierter Topos ist, der beispielsweise bei der Herrscherkritik zur Sprache kam, war dieser nicht direkt sexuell konnotiert. Historische Berichte schildern verschiedene grausame Herrscher, die Freude daran hatten, Verurteilte und in Ungnade gefallene Untergebene zu quälen, aber diese Qualen waren nicht sexualisiert. Beispielsweise erzählt das Zuozhuan von Herzog Ling von Jin, der von einem Turm seiner Residenz aus Pfeile auf Unbeteiligte abschoss und diese sogar tötete. Als ein Koch seine Bärentatzen - eine Spezialität - nicht vollständig gargekocht hatte, liess er ihn töten und die Frau des Chefkochs einen Korb mit dessen Leichnam abholen. In den historischen Aufzeichnungen gibt es zahlreiche solcher Geschichten; mit ihnen soll Verruchtheit eines Herrschers illustriert werden.

In den Berichten über Grausamkeiten sind die Subjekte auch nicht immer Männer. So lässt beispielsweise im Roman Die Drei Reiche die eben verwitwete Hauptfrau des Generals Yuan Shao sofort nach Bekanntwerden des Todes ihres Mannes dessen fünf Konkubinen nicht nur töten, sondern den Leichnamen auch die Haare abrasieren und die Gesichter zerkratzen, damit diese auch nicht im Jenseits nicht mit ihrem verstorbenen Mann Umgang pflegen. Manche Grausamkeiten wurden durchaus ebenfalls an Frauen praktiziert, aber auch hier ist festzustellen: In den Berichten gibt es keinerlei Hinweis auf eine Sexualisierung der Strafe, was sich schon daran zeigt, dass solche Grausamkeiten gemäss den Berichten in coram publico bei Hof vollzogen wurden. Es gibt insofern in der chinesischen Kulturtradition keine dem Marquis de Sade entsprechende Figur, die bekannt gewesen wäre für diese sexuelle Vorliebe.

Ebenso wenig gab es ein chinesisches Pendant zu Leopold Ritter von Sacher-Masoch, der in den Werken Don Juan von Kolomea (1866) und Venus im Pelz (1870) unterschiedliche Formen der körperlichen Liebe thematisierte, darunter den später so genannten Masochismus. 
170 Marc Winter: Lust und Schmerz als sexuelle Konzepte?

Eine vergleichbare Figur - historisch oder literarisch - ist in China nicht zu finden. Dass es keine Verbindung zwischen sexueller Lust und Schmerz gab, lässt sich auch an dem Paradebeispiel der eingebundenen Frauenfüsse in der späten Kaiserzeit, euphemistisch oft auch als «Lilienfüsse» bezeichnet, illustrieren. Die berüchtigte Fussverstümmelung, die im zweiten Jahrtausend des chinesischen Kaiserreiches zunächst von adeligen Frauen praktiziert wurde und eine immense Breitenwirkung entfaltete bis hin zu einem Massenphänomen, stellte ohne Zweifel ein tragisches Leiden für die betroffenen Frauen dar. Den Mädchen wurden ab dem fünften bis siebten Lebensjahr die Füsse eingebunden, so dass sie nicht mehr richtig wachsen konnten und verkrüppelten. Die erwachsenen Frauen hatten nur noch kleine Füsschen; das Ideal war eine Länge von drei Zoll, also zwischen acht und zehn Zentimeter. Solche Füsse galten als Schönheitsideal und wurden als sexueller Fetisch verehrt.

Die Verbindung zwischen dem sexuellen Fetisch, der Lust an kleinen Frauenfüssen, und dem unermesslichen Leiden der Mädchen, während ihre Füsse krankhaft verwuchsen, ist allerdings nicht nachzuweisen: Die Mädchen zogen ebenso wenig eine Befriedigung aus ihrem Schmerz wie ihre Mütter und Tanten, wenn sie sie ihnen dieselben Schmerzen auferlegten, die sie ebenfalls durchlitten hatten. Die Ethnologin Cilia Neumann hat herausgearbeitet, wie die Praxis des Füsseeinbindens weniger mit Sexualität oder mit der Beziehung zwischen Mann und Frau zusammenhing als vielmehr mit einem rite de passage, einer Kulthandlung, die den Übergang von der Kindheit zur Jugend markierte, den Eintritt ins Erwachsenenleben. ${ }^{10}$ Auch die Männer, die die kleinen Füsse als sexuellen Fetisch verehrte, zog keine Erregung aus dem Leiden der Mädchen, vielmehr wurde dieses ausgeblendet. Die Männer sahen die verkrüppelten Füsschen gar nicht nackt, weil sie in aufwändig bestickten dicken Socken steckten und so die Illusion bewahrt wurde, die Füsse seien so gewachsen.

10 Cilia Neumann: Lilienfüße in China. Aschenputtels Erbe, Frankfurt am Main 2016. 
171 Marc Winter: Lust und Schmerz als sexuelle Konzepte?

Im Folgenden soll ein Versuch unternommen werden, die Abwesenheit von Sadismus und Masochismus als sexuelle Spielarten in der vormodernen chinesischen Kultur zu erklären und die Frage beantwortet werden, wieso es keine schriftlichen Quellen und keine literarische Verarbeitung dieser sexuellen Praktiken gibt. Wieso wurden Masochismus und Sadismus kulturell nicht thematisiert? Wieso fanden sie in Sex-Handbüchern, die es in China seit mehr als zweitausend Jahren gab, keinen Niederschlag? Wieso wurden Sadismus und Masochismus in China - wie es scheint - seltener als in anderen Kulturen praktiziert.

Zunächst ist festzuhalten: Der chinesische Sexualdiskurs handelte von heterosexuellen Beziehungen zwischen Erwachsenen. Sexualität war in erster Linie und vor allem anderen ein Mittel zum Zweck der Erzeugung von Nachkommen. Es wurde als Pflicht aller Menschen aufgefasst, Kinder zu haben. Diese Pflicht erwuchs aus der Tatsache, dass man selbst Eltern hat und schloss die fundamentale Pflicht ein: die Pflicht der «Kindespietät», wie die konfuzianische Basistugend xiao 孝 in der Regel übersetzt wird. ${ }^{11}$

Sexualität wurde also in erster Linie im Kontext der Zeugung von Nachkommen thematisiert. Dementsprechend ging es in der Ratgeberliteratur wie den berühmten Kopfkissenbüchern weniger um maximale Erregung und grösstmögliche Befriedigung, sondern um die Möglichkeit, eine sozusagen garantierte Empfängnis herbeizuführen. In diesen Texten wird die Stimulierung der Frau thematisiert und auch, wie der Sexualpartner diese hervorrufen kann, aber dies jeweils klar im Kontext einer technischen Herbeiführung des Geschlechtsakts mit dem Ziel der Vereinigung der Körpersäfte von Mann und Frau und der Zeugung eines Kindes. Die Vorstellung war, dass die Körpersäfte beider Sexualpartner aus yinbeziehungsweise yang-Essenz bestehen, und dass das Zusammenfliessen der beiden Säfte das Kind zeugt. In der Sexualität wurde deshalb dem weiblichen Feuchtigkeitshaushalt eine wichtige Stellung zugesprochen.

11 Siehe auch den folgenden Abschnitt. 
172 Marc Winter: Lust und Schmerz als sexuelle Konzepte?

Der Sexualdiskurs beschränkte sich auf Heterosexualität; Homosexualität war eine Randerscheinung. ${ }^{12}$ Homoerotische Beziehungen waren weitgehend geduldet, das heisst, sie waren gesellschaftlich nicht geächtet, solange sie mit dem Plan, Kinder zu zeugen nicht in Konflikt gerieten. Die geringere Ablehnung von Homosexualität mag auch damit zusammenhängen, dass Geschlechtsidentitäten im vormodernen China nicht als so absolut angesehen wurden: Buddhas und Boddhisattvas änderten ihr Geschlecht, und die Grenzen zwischen Mann und Frau wurde im Vergleich zum Abendland als fliessend aufgefasst. Ein schwuler Mann war daher als eine Erscheinung im Spektrum geschlechtlicher Varianten und Möglichkeiten denkbar. Hinzu kommt, dass die Darstellung wechselseitiger Zuneigung zwischen engen Freunden in einem Masse toleriert wurde. Freundschaft, intellektuelle Kompatibilität und mögliche Sexualkontakte wurden als Einheit aufgefasst - als Beispiel seien die beiden Dichter Xi Kang und Yuan Ji aus dem 3. Jahrhundert genannt, zwei Dichter aus der Gruppe der «Sieben Weisen aus dem Bambushain», die sich nicht nur in dichterischer Hinsicht, sondern auch persönlich sehr nahestanden.

Wie in vormodernen patriarchalischen Gesellschaften üblich, wird auch in China weibliche Homosexualität begrüsst, solange sie keine Ausschliesslichkeit darstellt, aber sie wird weniger thematisiert. Frauen sollen mit Frauen verkehren, denn so tauschen sie Erfahrungen aus, die ihnen in dem Moment zugutekommen, wenn sie mit Männern Geschlechtsverkehr haben. Literarische Zeugnisse zu weiblicher Homosexualität sind daher seltener als solche zu homosexuellen Männern.

Sexualität wird grundsätzlich als etwas Normales, als natürliches Bedürfnis anerkannt, und sie darf und soll praktiziert werden.

Für homosexuelle Beziehungen unter Männern wurden Bezeichnungen verwendet wie «der geteilte Pfirsich» (分桃, fentao) oder «die Leidenschaft des abgeschnittenen Ärmels» (断袖之癖, duanxiu zhi pi). Erste Berichte gehen in die Zeit vor dem Kaiserreich zurück; so wird schon im Zhanguo Ce 戰國策 geschildert, wie ein General namens Jiang Wei 姜維 einen hübschen Jungen ins Lager der Gegner geschickt habe, um den Herrscher der Gegner zu verführen. 
Sexualität ist nichts «Sündiges», sondern ein Bedürfnis des gesunden Menschen. Dies gilt für verheiratete Paare, aber auch für Männer ohne Ehefrau, die ihre Bedürfnisse ins Bordell tragen konnten. Auch der Sex mit Prostituierten war gesellschaftlich akzeptiert, und er wird auch in der Literatur beschrieben. Sexualität wurde als natürliches Phänomen der Menschen aufgefasst, und Handbücher gaben Ratschläge, wie dieses Bedürfnis ausgelebt werden kann, um sexuelle Befriedigung zu erlangen und die Nachkommenschaft sicherzustellen. Auch wenn der Buddhismus - im Unterschied zum Christentum - Sexualität nicht als Sünde thematisiert, so ist der Einfluss der Religion in einem Aspekt bedeutsam: In China als weitgehend vom Buddhismus beeinflussten Land scheuten sich Menschen davor, anderen Menschen Schmerzen und Leiden zuzufügen, da Leid, das man anderen zufügt, karmisch auf einen selbst zurückfällt. Man musste also abwägen, ob man für den Lustgewinn karmische Schuld in Kauf nimmt.

Die Ausformung der Sexualität in einer Gesellschaft steht also in Wechselwirkung mit der herrschenden Religion, mit ihren Vorstellungen zur Körperlichkeit, zu den Geschlechtern und, wie Michel Foucault bemerkte, ihrem Verhältnis zur alles offenlegenden Wahrheit beziehungsweise zur Verschwiegenheit und zum Tabu. In seiner dreibändigen Studie zur Sexualität kommt Foucault auch auf Unterschiede zwischen Europa und aussereuropäischen Kulturen zu sprechen. Er beschreibt einen unterschiedlichen kulturellen Umgang mit Sexualität im Westen, wo sich eine scientia sexualis entwickelt habe, und in den Hochkulturen der islamischen Welt, Indiens und Ostasiens, die Sexualität als ars erotica verstehen und leben. Als entscheidend für diese divergente Entwicklung macht Foucault das Geständnis aus:

Das Geständnis war und ist bis heute die allgemeine Matrix, die die Produktion des wahren Diskurses über den Sex beherrscht. Allerdings hat es beträchtliche Transformationen erfahren. Für lange Zeit war es fest in die Praxis der Busse eingebaut. Nach und nach aber, mit dem Protestantismus, der Gegenreformation, der Pädagogik des 18. und der Medizin des 19. Jahrhunderts hat es seine rituelle und exklusive Lokalisierung verloren. [...] vor allem aber öffnet sich das Geständnis [...] neuen Methoden ihrer Erfassung. Es dreht sich nicht mehr nur darum zu sagen, was geschehen ist - der sexuelle Akt - und wie, sondern darum, in ihm und um ihn herum 
174 Marc Winter: Lust und Schmerz als sexuelle Konzepte?

die Gedanken zu rekonstruieren, die ihn verdoppelt haben, die Zwangsvorstellungen, die ihn begleiten, die Bilder, die Begehren, die Modulationen und die Qualität der Lust, denen er Raum gibt. Zweifellos zum ersten Mal hat eine Gesellschaft sich dazu herabgelassen, das Bekenntnis der individuellen Lüste anzuregen und anzuhören. ${ }^{13}$

Der Diskurs über Sexualität entwickelte sich im Westen also im Kontext von Ehe und Treue, von göttlichen Geboten und im Kontext der Erbsünde, einer Lehre des Augustinus, an der die römischkatholische Kirche unbeirrbar festhielt. Sünde, Beichte und Reue waren zentrale Momente, die einer Kultur der Selbsterforschung und dem Bekenntnis innerer Wünsche Vorschub leistete. Sexualität entwickelte sich entlang moralische Richtlinien als entweder gottgefällig und gut oder sündig und schlecht.

In China fand der Sexualdiskurs hingegen weniger im Kontext von Ehe, Treue und Moral statt, sondern im Kontext der Erhaltung der Gesundheit und sogar der Suche nach Unsterblichkeit. Es ging hier um anthropologische Konstanten, nicht um persönliche Vorlieben oder die Wünsche Einzelner oder die Frage nach moralischer Bewertung. Und hier liegt eine Erklärung, weshalb in China Lust und Schmerz nicht in einem sexuellen Zusammenhang erörtern werden. Der Geschlechtsakt wurde in China in den Zusammenhang des Ausgleichs der kosmischen Kräfte yin und yang eingeordnet. Harmonie und Ausgewogenheit waren anzustreben, sexuelle Erregung ohne sexuellen Höhepunkt wurde als gesundheitsfördernd angesehen, und der Körper war in der Lage, aus der Atemluft, der Lebensenergie qi, eine Essenz aus reinem yin oder yang herzustellen. Die Körpersäfte von Mann und Frau wurden auch beide mit der Bezeichnung jing, Lebensessenz bezeichnet. Diese komplementären Urkräfte werden bereits beim Küssen thematisch:

Diese beiden Elemente [yin und yang] wurden als entgegengesetzte kosmische Kräfte aufgefasst, und es wurde angenommen, dass die Sexualorgane elementare Kräfte besitzen. Allerdings [...] repräsentiert die Vulva nicht nur das yin und der Penis das yang, beide beziehen sich auf die untere Absorption. Die Münder und der Speichel von Mann und Frau sind ebenso

13 Michel Foucault: Sexualität und Wahrheit, I: Der Wille zum Wissen, übers. von Ulrich Raulf, Walter Seitter, Frankfurt am Main 1977, 81-82. 
175 Marc Winter: Lust und Schmerz als sexuelle Konzepte?

Organe mit elementaren Kräften, und diese repräsentieren die obere Absorption, die beim Küssen geschieht und die dadurch den Prozess vollendet. In einer Reihe von fortlaufenden sexuellen Sequenzen und Reaktionen würde eine wechselseitige Ergänzung der Geschlechter zu einem Zustand der Harmonie führen, in dem die Lebensessenz ( $q$ i) erhalten und das Leben verlängert würde. Gemäss derselben Logik würde ein Nichteinhalten dieser Abfolge zur Krankheit führen. Der Geschlechtsakt sollte daher in einer harmonischen und ruhigen Gemütsverfassung vollzogen werden. Daoistische Texte warnen daher generell vor heftigem oder gewalttätigem Verhalten, denn unpassender Verkehr in Hinsicht auf Geschwindigkeit oder Stellung würde zu körperlichen Beschwerden führen. Dies könnte erklären, wieso Sadomasochismus in der chinesischen Erotik nicht vorkommt und dafür ein harmonischer, kooperativer und verspielter Sex an seine Stelle getreten ist. ${ }^{14}$

Sex wird also als ein Weg zur Harmonisierung der kosmischen Kräfte im menschlichen Körper verstanden, dessen Ausübung einer Orthodoxie von Techniken folgt mit dem Ziel, den Körper gesund und im Gleichgewicht der kosmischen Kräfte zu halten.

Unsterblichkeit ist in dieser Hinsicht eine Extremform der Gesundheit. Sie war ein grosses Thema in China, denn ausgehend von der Beobachtung, dass der Mensch alt wird und schliesslich stirbt, suchte man nach den Gründen für das Altern, um Mittel und Wege,

These two elements were believed to be the polar forces of the cosmos, and the sex organs were believed to possess elemental forces. However, [...] the vulva not only represents the yin and the penis the yang, both of which refer to the lower absorption. The mouths and saliva of both male and female are also organs that have elemental forces, representing the upper absorption that happens during kissing and thereby completes the process. By following a number of sequential sexual progressions and reactions, a mutual supplement of each sex would lead to a state of harmony in which one's vital essence ( $q i)$ would be nourished and one's life would be prolonged. By the same logic, failure to adhere to the sequence would result in illness. Sexual engagement was to be conducted in a harmonious and tranquil mood. Daoist texts generally caution against sudden and violent sexual behavior because improper intercourse in terms of speed and posture would lead to physical afflictions. This may explain why sadomasochism is absent in Chinese erotica and is replaced instead by harmonious, cooperative, and playful sex. Eva Kit Wah Man: Kissing in Chinese Culture, in: Bodies in China. Philosophy, Aesthetics, Gender, and Politics, Hong Kong 2016, 73-84, hier 78-79. 
176 Marc Winter: Lust und Schmerz als sexuelle Konzepte?

dass die Menschen nicht alt werden und sterben. Den Grund für den Alterungsprozess sah man darin, dass die Menschen ihre Lebenskraft verlieren; als Gründe für den Verlust der Lebensenergie wurden unterschiedliche Faktoren angesehen: falsche Ernährung, Schwinden der kosmischen Energie im Körper, Unverträglichkeit des Individuums mit der kosmischen Konstellation und schliesslich Auszehrung durch Geschlechtsverkehr.

Als Mittel gegen das Altern und Sterben wurde in Texten ab dem 3. vorchristlichen Jahrhundert den Männern empfohlen, beim Sex keinen Orgasmus zu haben, denn der Orgasmus führe zum Verlust an Lebensenergie, die im Samen, dem lebensbringenden Körpersaft, offensichtlich vorhanden sei, weil zum einen Kinder daraus entstehen und zum anderen nach dem Höhepunkt Müdigkeit oder Erschöpfung einsetzen. Die Literatur kennt mehrere Beispiele von Personen, die zu intensiv dem Sex gefrönt haben und nachher nur noch ein Schatten ihrer selbst waren. Am berühmtesten ist vielleicht das Rou Putuan, das 1961 in der Schweiz wegen «Unzüchtigkeit» verboten wurde, wie der Verlag Die Waage anlässlich der Neuauflage 1995 mitteilte. ${ }^{15}$ In der Abteilung Sinologie des Ostasiatischen Seminars der Universität Zürich gab es in den 1980er Jahren ein Übersetzungsprojekt. Unterlagen zu diesem nicht erfolgreich zu Ende geführten Projekt existieren im Archiv des Instituts allerdings keine mehr. Einschlägig ist auch eine Passage im qingzeitlichen Gesellschaftsroman Hong Lou Meng, im deutschsprachigen Raum bekannt als Traum der Roten Kammer. Auch hier gibt sich ein Mann der Lust hin, wodurch seine Kräfte schwinden, bis er schliesslich an Auszehrung stirbt.

15 http://www.verlagdiewaage.ch/buecher/lesen/jouputuan.html Li Yu: Jou Pu Tuan. Ein erotisch-moralischer Roman aus der MingZeit (1633), übers. von Franz Kuhn, Zürich: Die Waage 1995. - 1. Aufl.: Hamburg: Die Waage 1965. 
177 Marc Winter: Lust und Schmerz als sexuelle Konzepte?

\section{Kindespietät}

Zwar lässt sich kein Zusammenhang zwischen sexueller Lust und Schmerz beziehungsweise Leiden nachweisen, wohl aber eine Verbindung zwischen Lust und Leiden im Kontext der konfuzianischen Kindespietät xiao 孝 im zweiten vorchristlichen Jahrhundert. Als Beweis der eigenen kindlichen Pietät war ein Leiden für das Wohl der Eltern willkommen und wurde von den pietätsvollen Kindern auch gerne in Kauf genommen, um die eigene Tugendhaftigkeit unter Beweis stellen zu können. Gemäss den Idealen der Pietät hatten Kinder ihren Eltern nicht nur zu gehorchen, sondern die Verfügungsgewalt der Eltern erstreckte sich auf den Körper des Nachwuchses, weshalb es in der späten Kaiserzeit als pietätslos galt, den eigenen Körper respektlos zu behandeln. So war es eine weit verbreitete Praxis, abgeschnittene Kopfhaare und Finger- oder Fussnägel zu sammeln und diese am Ende des Lebens zusammen mit dem Leichnam zu bestatten. Haare und Nägel wurden als Geschenk der Eltern betrachtet; ein achtloser Umgang damit galt daher als Beleidigung der Eltern. Mit dem Wiedererstarken des Konfuzianismus ab dem 14. Jahrhundert war Kindespietät eine zentrale soziale und kulturelle Praxis. Sie stützte sich nicht nur auf die Klassiker der Antike, sondern es entstanden neue Texte, die das gesellschaftliche Miteinander regelten.

Die Tugend der Kindespietät wurde als grundlegend für die gesellschaftliche Ordnung angesehen, und entsprechend mussten die Leistungen tugendhafter Kinder propagiert werden. Als Ikonen der Kindespietät galten demnach Kinder, die für das Wohl ihrer Eltern Leiden in Kauf nahmen. Hier gibt es tatsächlich einen Zusammenhang zwischen Lust und Leiden. In der einflussreichen Sammlung von 24 Beispielen für Kindespietät Ershisi Xiao 二十四孝 ist die Rede von Kindern, die grosses Leid auf sich nehmen, um ihren Eltern zu gefallen. Der Text, zwischen der zweiten Hälfte des 13. und der ersten Hälfte des 14. Jahrhundert von Guo Jujing 郭居敬 (1279-1368) redigiert oder verfasst, berichtet von 
Kindern, die für das Wohl ihrer Eltern erstaunliche Dinge durchlitten. ${ }^{16}$

Die Geschichte Zi wen bao xue (Den Mücken erlauben, sich am eigenen Blut zu sättigen) erzählt von einem Wu Meng, der seinen armen Eltern einen erholsamen Schlaf ermöglichte, indem er sich nackt den Bissen der Stechmücken aussetzte, weil seine Eltern sich kein Netz leisten konnten. Andere Geschichten thematisieren familiäre Konflikte. Wo bing qiu li (Aufs Eis liegen, um einen Karpfen zu fangen) erzählt von Wang Xiang, dessen Stiefmutter ihn schlug und die ihr leibliches Kind bevorzugte. Im Winter legte sich Wang Xiang ohne Kleider auf die Oberfläche eines zugefrorenen Sees, um so ein Loch in die Oberfläche zu schmelzen, damit er für die Stiefmutter einen Karpfen fischen konnte.

Andere Geschichten erzählen von Opfern oder Taten. In Chang fen you xin (Mit sorgenerfülltem Herz die Exkremente des Vaters kosten) vernachlässigte Yu Qianlou seine Kindespflicht, indem er an einem entfernten Ort einen Beamtenposten annahm. Als sein Vater erkrankte, eilte er nach Hause, wo ihm der Arzt mitteilte, dass die Wirksamkeit der verabreichten Medizin nur eingeschätzt werden könne, wenn man die Exkremente des Vaters auf ihren Geschmack hin prüfe, was der Sohn dann pflichtschuldigst tat. In $E$ hu jiu qin (Den Tiger würgen, um den Vater zu retten) überwand das Mädchen Yang Xiang ihre natürliche Furcht und verteidigte ihren Vater vor einem Tigerangriff, indem sie auf die Raubkatze sprang und diese würgte, bis sie vom Vater abliess. Danach trug sie den verletzten Vater nach Hause.

Solche Geschichten, die sich in China auch noch heute grosser Beliebtheit erfreuen, stellen Kindespietät als eine Leistung dar, die vielleicht im Moment Leiden verursachen kann, deren Lohn - in

16 Die Beispiele entstammen alle der modernen illustrierten Neuausgabe nach Ren Bonian 任伯年 et al. (Hg.): Ershisi xiao tu 二十四孝图 (Illustrierte Ausgabe der 24 Geschichten zur Kindespietät), Tianjin 2009. Ren Bonian illustrierte die Geschichten erst in der Qingzeit, er war kein Zeitgenosse von Guo Jujing. Vgl. hierzu auch M. Winter: «Konfuzianismus», in: Enzyklopädie des Märchens. Handwörterbuch zur historischen und vergleichenden Erzählforschung, XIV / 4, Berlin, Boston 2014, 1730-1734. 
Form von Anerkennung durch die Eltern und die Gesellschaft aber diese Leiden wettmacht. Die Geschichten bleiben interessant wegen der zuweilen bizarren Ideen. In Wen lei qi mu (Den Donner hören und am Grab weinen) wird erzählt, wie ein gewisser Wang Pou seine schreckhafte Mutter nicht nur zeitlebens bei jedem Gewitter tröstete und dafür von wo auch immer nach Hause lief. Nach ihrem Tod behält er diese Sorge auch für ihr Grab bei. Nicht einmal der Einmarsch feindlicher Truppen konnte ihn dazu bewegen, sich vom Grab der Mutter zu entfernen. Dabei verlor er zwar sein Leben, aber er blieb als vorbildliche Verkörperung der Kindespietät in Erinnerung. Die Geschichte Wei mu mai er (Zugunsten der Mutter den Sohn begraben) schliesslich erzählt von einem Drei-Generationen-Haushalt in der Übergangszeit zwischen zwei Dynastien, meist Phasen der Unsicherheit und der finanziellen Not. Die Familie war arm, und als die Grossmutter Hunger litt, sah ihr Sohn nur den Ausweg, seinen einzigen Sohn zu töten, um auf diese Weise mehr Nahrung zur Verfügung zu haben. Sein Argument lautete: «Kinder können wir immer noch bekommen, eine Mutter gibt es im Leben dagegen nur einmal.» Als die Eltern vor der Tat das Grab für ihren Sohn ausheben, stossen sie auf einen Sack voller Goldstücke, so dass ihre Probleme gelöst waren.

In diesen Geschichten wird das meist körperliche Leiden der Kinder belohnt: zufriedene Eltern, hohe gesellschaftliche Anerkennung für das Kind wie auch für die Eltern, die ein solch tugendhaftes Kind grossgezogen haben. Dieses aus dem Leiden gewonnene Ansehen, im chinesischen Kontext oftmals als «Gesicht» bezeichnet, erfüllt das Kind mit Stolz und wiegt das Leiden auf. Man kann hierin durchaus einen «Lustgewinn» sehen, weil das Kind schon in der Leidensphase weiss, dass nach überstandenem Martyrium die Freude umso grösser sein wird. Menschen erleiden - zuweilen «lustvoll»Schmerzen um eines höheren Zweckes willen. Ihr Leiden verschafft ihnen die «Lust», als Vertreter der Kindespietät die Verehrung und Zuneigung ihrer Eltern und der Gesellschaft zu erlangen. 
180 Marc Winter: Lust und Schmerz als sexuelle Konzepte?

conexus 1 (2018) 161-180

(C) 2018 Marc Winter. Dieser Beitrag darf im Rahmen der Lizenz CC BYNC-ND 4.0 - Creative Commons: Namensnennung/nicht kommerziell/ keine Bearbeitungen - weiterverbreitet werden.

\section{(c) (1) (8) 9}

https://doi.org/10.24445/ conexus.2018.01.011

PD Dr. Marc Winter, Universität Zürich, Asien-Orient-Institut, Zürichbergstrasse 4, 8032 Zürich

marc.winter@aoi.uzh.ch 